\title{
DNA Methylation Leads to DNA Repair Gene Down-Regulation and Trinucleotide Repeat Expansion in Patient-Derived Huntington Disease Cells
}

\author{
Peter A. Mollica, ${ }^{\star \dagger}$ John A. Reid, ${ }^{\dagger}$ Roy C. Ogle, ${ }^{\dagger}$ Patrick C. Sachs, ${ }^{\dagger}$ and Robert D. Bruno
}

From the Departments of Biological Sciences, * Medical Diagnostics and Translational Sciences, ${ }^{\dagger}$ and Engineering and Technology, ${ }^{\ddagger}$ Old Dominion University, Norfolk, Virginia

\author{
Accepted for publication \\ March 16, 2016. \\ Address correspondence to \\ Robert D. Bruno, Ph.D., or \\ Patrick C. Sachs, Ph.D., 4211 \\ Monarch Way, Suite 365, \\ Norfolk, VA 23529. E-mail: \\ rbruno@odu.edu or psachs@ \\ odu.edu.
}

\begin{abstract}
Huntington disease (HD) is an autosomal dominantly inherited disease that exhibits genetic anticipation of affected progeny due to expansions of a trinucleotide repeat (TNR) region within the HTT gene. DNA repair machinery is a known effector of TNR instability; however, the specific defects in HD cells that lead to TNR expansion are unknown. We hypothesized that HD cells would be deficient in DNA repair gene expression. To test this hypothesis, we analyzed expression of select DNA repair genes involved in mismatch/loop-out repair (APEX1, BRCA1, RPA1, and RPA3) in patient-derived HD cells and found each was consistently down-regulated relative to wild-type samples taken from unaffected individuals in the same family. Rescue of DNA repair gene expression by 5-azacytidine treatment identified DNA methylation as a mediator of DNA repair gene expression deficiency. Bisulfite sequencing confirmed hypermethylation of the APEX1 promoter region in HD cells relative to control, as well as 5-azacytidine-induced hypomethylation. 5-Azacytidine treatments also resulted in stabilization of TNR expansion within the mutant $H T T$ allele during long-term culture of HD cells. Our findings indicate that DNA methylation leads to DNA repair down-regulation and TNR instability in mitotically active HD cells and offer a proof of principle that epigenetic interventions can curb TNR expansions. (Am J Pathol 2016, 186: 1967-1976; http://dx.doi.org/10.1016/j.ajpath.2016.03.014)
\end{abstract}

Huntington disease (HD) is a neurodegenerative disease that affects medium spiny neurons in the basal ganglia, resulting in involuntary muscle movements and ultimately leading to death. It is caused by an expansion of a cytosine-adenineguanine (CAG) trinucleotide repeat (TNR) region in exon 1 of the HTT gene. ${ }^{1} \mathrm{HD}$ is a dynamic mutation disease in which the number of TNRs increases the probability of expression of the mutant phenotype. The disease does not affect individuals with $<35$ TNRs, but individuals with 35 to 40 repeats are at an increased risk of developing HD. Expansions $>40$ repeats inevitably lead to HD progression. ${ }^{2}$ Although the mechanism is not fully understood, HDaffected individuals exhibit genetic anticipation caused by subsequent generations of HD-affected individuals developing expanded versions of the mutated allele. ${ }^{3-5}$ Increased expansions in successive generations correlate with more severe symptomatic expression and earlier age at onset in
$70 \%$ of individuals. ${ }^{6,7}$ Interestingly, postmortem analysis of affected neuronal cells and other somatic tissues in HD mouse models reveals disease-causing gene CAG mosaicism, in which CAG repeats of affected alleles in different tissues have different sized expansions. ${ }^{8-11}$ In addition, a myriad of TNR instability effectors have been identified, such as environmental stresses, chemical inducers, and DNA repair gene expression. ${ }^{12-15}$ This evidence infers that TNR instability is present not only in germline cells but also throughout development and maturation and thus dependent on efficacy of DNA repair and its ability to appropriately respond to endogenous and exogenous DNA-damaging

Supported by institutional start-up support from the College of Health Sciences at Old Dominion University (R.D.B. and P.C.S.).

P.C.S. and R.D.B. contributed equally to this work as senior authors.

Disclosures: None declared. 
agents and modifiers. Furthermore, emerging data have recently implicated TNR instability in replicating neuronal support cells as possible mediators of disease severity and progression. ${ }^{16-18}$ Thus, deciphering how TNR expansions occur during development and in proliferating cell populations during development and adulthood is vital to our understanding of the pathogenesis of HD. These data would lead to therapeutic strategies aimed at preventing TNR expansions and delaying the onset and severity of HD symptoms in patients inheriting a mutated HTT allele.

Unfortunately, the comprehensive mechanism of TNR instability eludes researchers. Of the proposed mechanisms, heteroduplex formation and the inability for successful resolution during replication are the common factors behind TNR instability. ${ }^{19}$ Through intrastand complementary base pairing, single-stranded DNA generates secondary structures, such as hairpins or loop-outs. ${ }^{20}$ Although this secondary structure meets the criteria for DNA damage, the lesion is ignored in HD cells, and the expanded allele generally results in the dissemination of a larger CAG repeat region to daughter cells.

The inability of DNA repair machinery to stabilize CAG repeats in HD cells led us to hypothesize that HD somatic tissues have abnormal expression of DNA repair genes, sensitizing them to CAG TNR expansions. To test this hypothesis, we analyzed DNA repair gene expression in fibroblasts from HD patients and wild-type controls. Because TNR instability is seen in all tissue types, we chose to look at fibroblasts because they are mitotically active and thus would better represent TNR expansions occurring during development. We determined that key DNA repair genes were down-regulated in HD fibroblasts compared with wild-type controls and that the promoter region of the down-regulated repair gene, APEX1, was hypermethylated compared with controls. Treatment with the DNA methyltransferase inhibitor 5-azacytidine resulted in increased DNA repair expression and marked stabilization of CAG repeats during long-term culture. This is the first study to identify endogenously deficient DNA repair genes in human $\mathrm{HD}$ fibroblasts and is proof of principle that pharmacologic intervention can lead to increased TNR stability in patients at risk for the disease.

\section{Materials and Methods}

\section{Cell Lines and Cell Culture}

Three previously confirmed HD (GM03621, GM02079, and GM03868), two wild-type (GM02187 and GM02153), and two unknown (GM02191 and GM04022) fibroblast cell lines were obtained from Coriell Cell Repositories (Camden, NJ). HD status of cell lines used were confirmed through the Coriell Cell Repositories online database. Unknown fibroblast samples were obtained from donors of whom one parent was confirmed to be affected by HD but remained undiagnosed. Genetic characteristics of exon 1 of the HTT gene of each cell line used in our experiments are detailed in Table 1. Cell lines were cultured and maintained at $37^{\circ} \mathrm{C}$ in a humidified incubator with $5 \%$ $\mathrm{CO}_{2}$. Fibroblasts were cultured in Dulbecco's modified Eagle's medium with glutamax (Life Technologies, Carlsbad, CA) with $10 \%$ fetal bovine serum (Life Technologies) as recommended by Coriell Cell Repositories. Cell media also contained $1.0 \%$ antibioticantimycotic $(100 \times)$ (Life Technologies). Cells were seeded in $\mathrm{T} 75-\mathrm{cm}^{2}$ flasks at $5.0 \times 10^{4}$ cells, and medium was replenished between 72 and 96 hours of seeding. Cells were passaged every 5 to 6 days using TrypLE Express (Life Technologies) following manufacturer's instructions.

\section{RNA and Genomic DNA Isolation}

Total RNA was isolated from cells on growth to $70 \%$ to $80 \%$ confluency (approximately 1 million cells) with Trizol (Thermo Fisher, Waltham, MA) following the manufacturer's protocol. Approximately $1.0 \times 10^{6}$ cells were harvested, and genomic DNA (gDNA) was extracted using DNeasy Blood \& Tissue Kit (Qiagen, Hilden, Germany) according to the manufacturer's protocol. Samples were

Table 1 Genetic Characteristics of Exon 1 of the HTT Gene of Each Cell Line Used in This Study

\begin{tabular}{lllllll}
\hline Cell line & Experiment designation & Age, years & Reported status & Reported CAG $_{n}$ & Confirmed status & Confirmed CAG \\
\hline GM02187 & Wt-1 & 60 & Wild type & $17-23$ & Wild type & $17-23$ \\
GM02153 & Wt-2 & 40 & Wild type & $16-32$ & Wild type \\
GM02079 & HD-1 & 48 & HD & $21-44$ & HD & $16-32$ \\
GM03621 & HD-2 & 29 & HD & $18-60$ & HD & $21-45$ \\
GM03868 & HD-3 & 45 & HD & $18-44$ & HD & $18-60$ \\
GM04022 & pHD-1 & 28 & Unknown & - & HD & $18-48$ \\
GM02191 & pHD-2 & 32 & Unknown & - & HD & $18-44$ \\
\hline
\end{tabular}

HD-affected and wild-type fibroblast samples are all female. All cell lines were purchased from the Coriell Cell Repositories. The status of fibroblast lines GM02191 and GM04022 were designated unknown on ordering and initial experiments because they had not yet been characterized and were subsequently confirmed to be HD-affected through capillary electrophoresis fragment analysis. Experimental designation is the abbreviation used throughout the article. The CAG expansions shown above represent the characterizations previously published by Coriell Cell Repositories, with the exception of our unknown samples (GM04022 and GM02191) in which the CAG is the first characterization by fragment analysis completed.

CAG, cytosine-adenine-guanine; HD, Huntington disease; pHD, potential Huntington disease; Wt, wild type. 
concentrated by ethanol precipitation when needed. RNA and DNA quantity and purity were assessed by the absorbance at 260/280 nm method using a NanoDrop 2000 (Thermo Fisher), with 260/280 ratios of approximately 2.0 and 260/230 ratios slightly higher than 1.8 .

\section{5-Azacytidine and Trichostatin-A Treatment}

For gene and protein expression assays, cell medium was supplemented with 5-azacytidine diluted in phosphate buffer solution to achieve $10-\mu \mathrm{mol} / \mathrm{L}$ concentrations for 120 hours. The $10-\mu \mathrm{mol} / \mathrm{L}$ dosage was the predetermined $50 \%$ lethal concentration of 5-azacytidine (data not shown). For TNR stability assays, cells were treated intermittently with $10 \mu \mathrm{mol} / \mathrm{L} 5$-azacytidine during 35 days to reach four population doublings. Treatment schedule was as follows: pass in normal medium for 24 hours, treat with $10 \mu \mathrm{mol} / \mathrm{L} \mathrm{5-}$ azacytidine for 5 days, allow cells to recover for 3 to 5 days in normal medium, repeat with passaging at a 1:2 split ratio. During 5-azacytidne treatment days, media was replaced daily with freshly prepared 5-azacytidine to optimize passive demethylation during DNA synthesis and to facilitate the removal of toxic products. gDNA samples were collected at the beginning of each treatment schedule and at the end of the designated time frame.

For histone-deacetylase inhibition treatment, complete fibroblast medium was supplemented with $0.5 \mu \mathrm{mol} / \mathrm{L}$ and $5.0 \mu \mathrm{mol} / \mathrm{L}$ trichostatin-A (TSA) (Sigma-Aldrich, St. Louis, MO) reconstituted in dimethyl sulfoxide to achieve $0.01 \%$ $\mathrm{v} / \mathrm{v}$. TSA that contained media was applied 16 hours after passaging cells and allowed to remain for 24 hours. On removal of TSA, total RNA was immediately extracted.

\section{Quantitative RT-PCR}

One microgram of each RNA sample was reverse transcribed into cDNA using a High Capacity cDNA Reverse Transcription Kit (Applied Biosystems, Foster City, CA) according to the manufacturer's instructions. We used TaqMan Gene Expression Assays (Applied Biosystems) to assess mRNA levels. TaqMan Gene Expression Assays provided optimized primers and probes specific for DNA repair genes APEXI (Hs00959050_g1), BRCAl (Hs01556193_m1), RPAl (Hs00161419_m1), and RPA3 (Hs01047933_g1) and the endogenous housekeeping gene ACTB (Hs99999903_m1). Quantitative RT-PCR (RT-qPCR) experiments were conducted with a StepOnePlus Real-Time PCR System (Applied Biosystems) using TaqMan Fast Advanced Master Mix (Life Technologies) using $50 \mathrm{ng}$ of cDNA from each sample per the manufacturer's protocol. All experiment reactions were independently completed in triplicate. Negative template controls were run on each experiment containing nuclease-free water. All cell lines were tested three independent times, with at least two population doubling events occurring between sample experiments. Each experimental sample was also subsampled three times during RT-qPCR reactions. Relative fold-changes were calculated using the $2^{-\Delta \Delta \mathrm{Ct}}$ method. Significance was determined by comparing the $2^{-\Delta \mathrm{Ct}}$ value using a one-way analysis of variance with a Dunnett's Post Hoc Test.

\section{Immunofluorescence of DNA Repair Genes}

Cell samples were seeded onto Lab-Tek microscope chamber slides at a density of $1.5 \times 10^{3}$ cells $/ \mathrm{mL}$ and fixed with $10 \%$ formalin for 30 minutes at room temperature. Samples were blocked with $10 \%$ goat serum for 60 minutes at room temperature and then incubated with primary antibody overnight at $4^{\circ} \mathrm{C}$ in a humidified chamber. Primary antibodies were obtained from ABCAM (Cambridge, MA): APEX1 antibody (ab105081; 1:700), BRCA1 antibody (ab16781; 1:250), RPA1 antibody (ab79398; 1:250), and RPA3 antibody (ab6432; 1:50). Samples were then incubated with Alexa Fluor 488 conjugated secondary antirabbit or anti-mouse antibodies (Thermo Fisher, 1:1000) for 60 minutes. Slides were counterstained with DAPI. Fluorescent images were acquired with a Zeiss Axio Observer.Z1 microscope (Carl Zeiss AG, Jena, Germany) at $10 \times$ objective. Images were collected in 16-bit black and white to maximize resolution. False coloring was added by ImageJ software version 1.50e FIJI (NIH, Bethesda, MD; http://imagej.nih.gov/ij). At least three images of each gene and cell line were collected to ensure global capture of the population of cells. Relative quantitation was also conducted using ImageJ. Cell fluorescence was determined by multiplying the total area of the image by mean background of the image, then subtracted from integrated density. The cell fluorescence of each image was then divided by the number of complete nuclei in the image to give the corrected total cell fluorescence (CTCF). Mean CTCF is represented graphically as normalized values to the mean CTCF of wild-type fibroblast sample. For statistical analysis, relative fold-changes were compared using the Wilcoxon rank sum test.

\section{Triplet Repeat Primed PCR}

Primers were synthesized by Integrated DNA Technologies (Coralville, IA). The forward primer sequence was 5'-ATGAAGGCCTTCGAGTCCCTCAAGTC-3', with a carboxyfluorescein at its $5^{\prime}$ end, and the reverse was $5^{\prime}$-CGGTGGCGGCTGTTGCTGCTGCTGCTGCTG- $3^{\prime}{ }^{21}$ Amplification reactions were run in $20 \mu \mathrm{L}$ volumes and contained the following components; 1× FailSafe Premix J (Epicenter, Madison, WI), $1 \mathrm{U}$ of Platinum Taq DNA Polymerase High Fidelity (Life Technologies), $1.0 \mu \mathrm{L}$ of 20 to $25 \mathrm{ng} / \mu \mathrm{L}$ of gDNA sample, and $0.5 \mu \mathrm{mol} / \mathrm{L}$ each primer. PCR conditions were as follows: $95.0^{\circ} \mathrm{C}$ for 1 minute hot start/initial denaturation, followed by 35 cycles of $94.0^{\circ} \mathrm{C}$ for 1 minute, $64.0^{\circ} \mathrm{C}$ for 1 minute, $72.0^{\circ} \mathrm{C}$ for 2 minutes, and a final extension of 15 minutes at $72.0^{\circ} \mathrm{C}$. One microliter of each sample was combined with $1.0 \mu \mathrm{L}$ of Mapmarker 1000 internal size standard (BioVentures Inc., Murfreesboro, 
$\mathrm{TN}$ ) and $9.0 \mu \mathrm{L}$ of Hi-Di Formamide (Life Technologies). Just before sample loading of $10 \mu \mathrm{L}$ onto capillary electrophoresis plates, each sample was heated to $95.0^{\circ} \mathrm{C}$ for 2 minutes for denaturation and then immediately placed on ice for 5 minutes. Capillary electrophoresis fragment analysis was conducted on an Applied Biosystems 3130 Genetic Analyzer (Applied Biosystems) with 36-cm arrays and POP-7 Polymer-CG (Applied Biosystems). Electrokinetic injection of samples was initiated with $12 \mathrm{kV}$ for 16 seconds and then electrophoresed at $15 \mathrm{kV}$ for $1200 \mathrm{sec}-$ onds at $60^{\circ} \mathrm{C}$. Raw data were analyzed using GeneMarker software version 2.6.4 (SoftGenetics LLC, State College, PA). ${ }^{21} \mathrm{We}$ conducted amplified fragment length polymorphism analysis using optimized macros for TNR expansion calling and labeling. Macros for bin calling were customized using gDNA samples obtained from Coriell Cell Repositories and were graciously created by Mohamed Jama (University of Utah). As positive controls, we obtained 6 gDNA samples from various HD-affected and wild-type characterizations. These chosen positive control gDNA samples had previously been tested in multiple laboratories and were sequenced to confirm CAG repeats (P.A.M., unpublished data). ${ }^{21}$

\section{Bisulfite Conversion and meCpG Analysis}

The gDNA samples were treated to bisulfite conversion using the EpiTect Fast Bisulfite Kit (Qiagen) according to the manufacturer's protocol. Primers were designed to evaluate methylation patterns approximately $-500 \mathrm{bp}$ upstream of APEX1 using MethPrimer online software. ${ }^{22}$ The region was amplified in two segments: forward: 5'-ATAATGTGTTGTGTATTTGGTATAA-3', reverse: 5'-ATAACAACCTAACTCCTCCTAACCC-3'; forward: 5' ${ }^{\prime}$-TGTTAGGAGTTGTGGAGGTTTTTAT- $3^{\prime}$, reverse: $5^{\prime}$-TTCTCTAAAACACTTAAATCCCCAA- ${ }^{\prime}$. PCR amplification of $500 \mathrm{ng}$ of bisulfite-treated gDNA was conducted using $1 \mathrm{U}$ of Platinum Taq DNA Polymerase High Fidelity (Life Technologies) as previously described in Materials and Methods. We used a touch-up PCR cycling protocol where the annealing temperature was gradually increased after the first and second set of five cycles, with annealing temperatures increasing from $53^{\circ} \mathrm{C}$ to $56^{\circ} \mathrm{C}$ and then finally $60^{\circ} \mathrm{C}$ for the remaining 30 cycles. PCR cycling conditions were as follows: $95^{\circ} \mathrm{C}$ for 1 -minute hot start, followed by a touch-up protocol with increasing annealing temperatures for 1 minute, and $72^{\circ} \mathrm{C}$ for 1 minute for 40 total cycles. A final extension of 10 minutes at $72^{\circ} \mathrm{C}$ was applied at the end. Correct amplicons were assessed using gel electrophoresis on a $1.5 \%$ agarose gel. Appropriate bands were extracted using QIAquick Gel Extraction Kit (Qiagen). Each extracted amplicon was then inserted into the pCR4-TOPO TA vector with the TOPO TA cloning kit for sequencing (Thermo Fisher) per the manufacturer's protocol. Cycle sequencing of fragments was conducted using the M13 forward and M13 reverse primer sets provided in the TOPO TA kit. Methylation analysis and representation were conducted using Methylation Plotter online software. ${ }^{23}$

\section{Results}

APEX1, BRCA1, RPA1, and RPA3 Gene Expression Is Deficient in Both HD and Unknown Fibroblasts

To determine whether DNA repair gene expression was perturbed in proliferating HD cells, we performed RT-qPCR to detect relative mRNA expression level of four DNA repair genes (APEXI, BRCA1, RPA1, and RPA3) in HD samples. We compared fibroblasts isolated from three confirmed HD patients (GM02079, GM03621, GM03868), two wild-type patients (GM02187 and GM02153), and two unknown patients (GM02079 and GM04022) who had a $50 \%$ chance of inheriting the mutant $H T T$ allele. Heretofore, confirmed HD cell lines are designated as HD-n, unknowns are designated as potential HD pHD-n, and wild-type samples designated Wt-n (Table 1). We chose to examine $A P E X 1, B R C A 1, R P A 1$, and RPA3 because they are effectors of TNR instability-induced DNA damage and because we had previously observed their down-regulation in HD cells (unpublished data). ${ }^{24-29}$ Our analysis revealed statistically significant down-regulation of all four genes in all HD-affected and unknown fibroblast lines (Figure 1). The mRNA levels presented are in relation to control sample Wt-1 because this sample had lower overall expression of the four DNA repair genes when compared with the Wt-2 sample and thus represents a more stringent comparison.

\section{Unknown Samples Are Affected by mHTT Expansion}

Because both of our unknown samples (pHD-1 and pHD-2) had significant down-regulation of all four DNA repair genes, we sought to identify if they did, in fact, have an expanded HTT allele. To determine HD status of unknown fibroblast lines, we conducted capillary electrophoresis fragment analysis using triplet repeat primed PCR on all samples (Table 1 and Supplemental Figure S1). Both unknown fibroblast lines pHD-1 and pHD-2 were confirmed HD, with alleles that contained CAG repeats of 18/44 and 17/42, respectively (Supplemental Figure S1, A and B). Combined with data identifying APEXI, BRCAl, RPAl, and $R P A 3$ down-regulation, this confirmation of unknown samples being affected by HD identifies therapeutic biomarkers, as well as a possible alternate means for HD diagnosis.

Interestingly, we witnessed TNR instability in previously characterized HD samples HD-1 and HD-3, whereas HD-2 remained static (Supplemental Figure S1C, Supplemental Figure S2, A and B, and Table 1). Previously, Coriell Cell Repositories reported HTT gene characterization of HD-1 as containing 21/44 CAG repeats and HD-3 as containing 18/44 CAG repeats. Our initial characterization was 


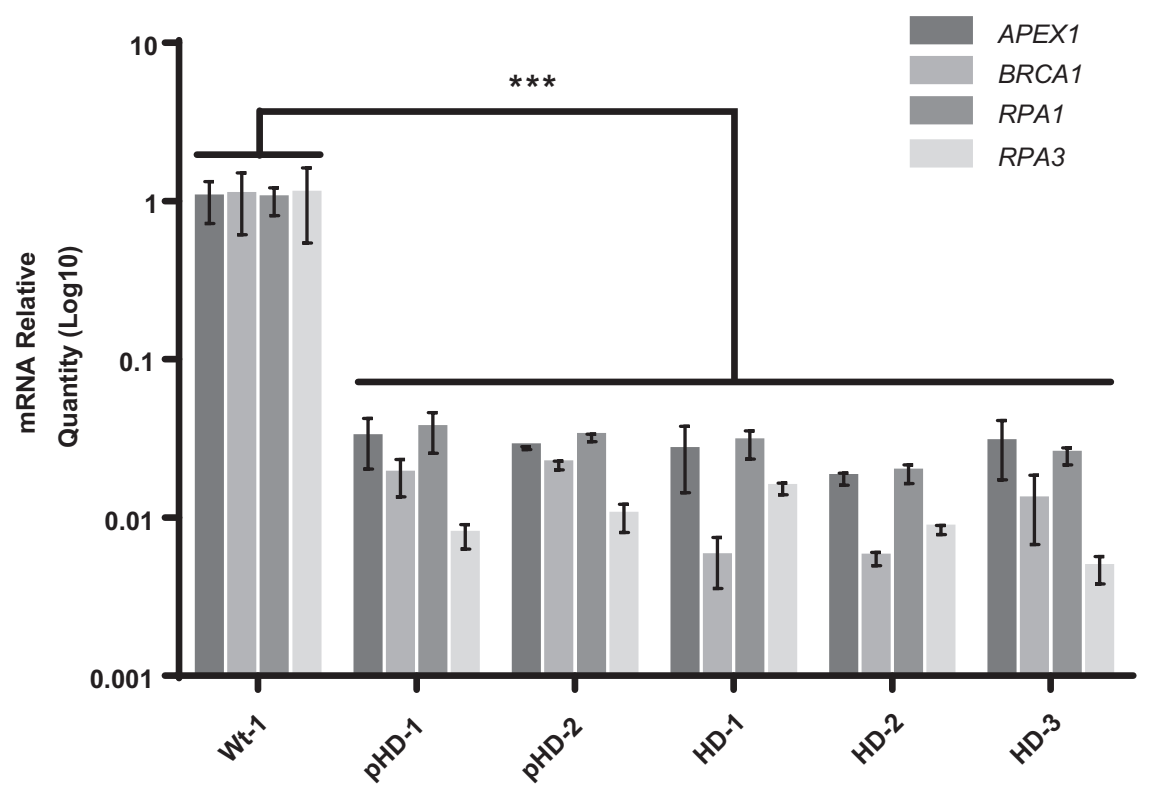

Figure 1 DNA repair deficiencies in multiple Huntington disease (HD) fibroblast cell samples. APEX1, BRCA1, RPA1, and RPA3 expression in wildtype (Wt-1), potential HD-affected (pHD), and HDaffected (HD) fibroblasts. Using TaqMan Gene Expression Assays, we identified HD DNA repair mRNA down-regulation of APEX1, BRCA1, RPA1, and $R P A 3$. Gene expression is determined by the $\left(2^{-\Delta \Delta \mathrm{Ct}}\right)$ of the mean of each independent triplicate. Error bars represent the SD of the mean between replicate samples. Each tested gene shows significant decreases in expression for all $\mathrm{HD}$ and $\mathrm{pHD}$ cell lines, relative to the Wt sample. Gene expression is normalized to the Wt-1 fibroblast line GM02187 gene expression. Statistical significance was determined using a two-tailed, homoscedastic $t$-test. ${ }^{* * *} P<0.001$. conducted after 10 population doublings and revealed HTT expansions in the mutated allele to 45 and 48 , respectively. Interestingly, HD-3 had a contraction in the unaffected allele from previously reported 18 to 17 . No instability was detected in either wild-types samples (Wt-1 or Wt-2) after identical population doublings (Supplemental Figure S2C, Supplemental Figure S3, and Table 1).

\section{APEX1, BRCA1, RPA1, and RPA3 Protein Levels Are Down-Regulated in HD Fibroblasts}

To determine whether protein levels of DNA repair genes matched our findings from our mRNA analysis, we conducted immunocytochemistry of all fibroblast lines (Figure 2A). CTCF revealed that protein levels in pHD-1 and pHD-2 samples were approximately $23 \%$ of APEX1, $22 \%$ of BRCA1, $8 \%$ of RPA1, and $32 \%$ of RPA3 (Figure $2 \mathrm{~B}$ ) and $54 \%$ of APEX1, 35\% of BRCA1, $46 \%$ of RPA1, and $36 \%$ of RPA3 (Figure 2C), respectively. Protein levels are relative to the lowest-expressing wild-type fibroblast sample (Wt-1). These data indicate that DNA repair components are significantly decreased in HD fibroblast samples, possibly having consequences on DNA repair pathways to efficiently address lesions and adducts generated during DNA synthesis.

\section{APEX1, BRCA1, and RPA1 Gene Expression Is Affected by 5 -Azacytidine Treatments}

We next wanted to identify the potential mechanism of the observed DNA repair gene down-regulation. Because no previous DNA repair gene mutational events have been associated with HD, we hypothesized that epigenetic regulators could be a primary cause of the observed downregulations. To test for the involvement of histone acetylation and DNA condensation as a primary regulator, we treated pHD-1 and pHD-2 samples with $5 \mu \mathrm{mol} / \mathrm{L}$ TSA, a potent global deacetylase inhibitor, for 24 hours. RT-qPCR revealed no significant changes in DNA repair gene expression in either pHD-1 (Figure 3A) or pHD-2 (Figure 3B) in response to TSA treatments, suggesting hyperacetylation of histones was not primarily responsible for the suppression of DNA repair genes.

Next, we chose to investigate the effects of 5-azacytidine to determine possible involvement of DNA methylation in DNA repair gene down-regulation. pHD-1 and pHD-2 samples were seeded and cultured in the presence of culture media supplemented with $10 \mu \mathrm{mol} / \mathrm{L}$ 5-azacytidine for 120 hours. After treatment, pHD-1 and pHD-2 revealed significant up-regulation of APEX1, BRCA1, and RPA1 mRNA as confirmed by RT-qPCR (Figure 3, C and D) and protein expression as measured by immunocytochemistry (Supplemental Figure S4 and Figure 3, E and F). Overall, these data suggest that the DNA repair gene expression in HD fibroblasts was at least partially restored by treatment with 5-azacytidine.

\section{Differential Methylation Patterning in APEX1 Promoter in HD Fibroblasts}

To determine whether DNA methylation was increased near promoter regions of DNA repair genes in HD cells and if this was reversed by 5-azacytadine treatment, we conducted bisulfite sequencing analysis of approximately 500 bp upstream to approximately 75 bases downstream of the APEX1 transcriptional start site. We compared both untreated and 5-azacytadine treated Wt-1, pHD-1, and pHD-2 samples. Bisulfite sequencing revealed that no methylated $\mathrm{CpG}$ sites were present in treated $\mathrm{Wt}-1$ and untreated $\mathrm{Wt}-1$ samples in the analyzed region (Figure 4). In contrast, untreated HD samples had methylated $\mathrm{CpG}$ sites in the $A P E X 1$ promoter 
A

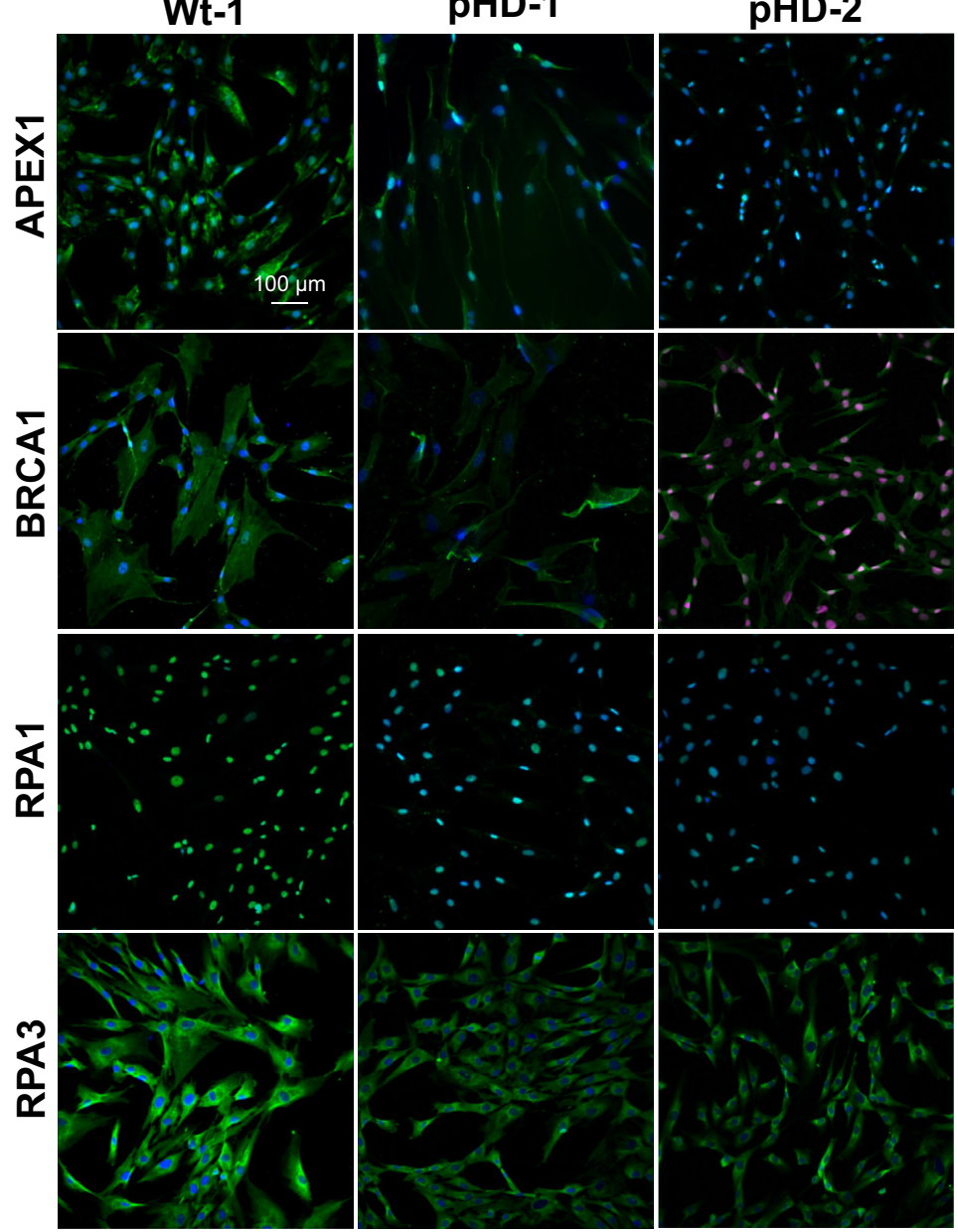

B

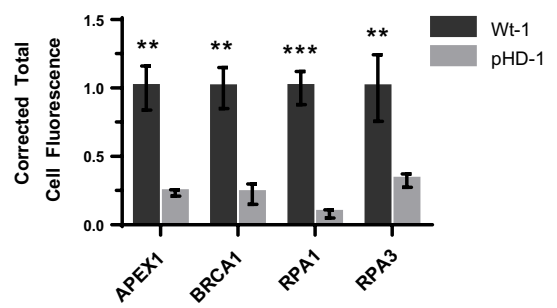

C

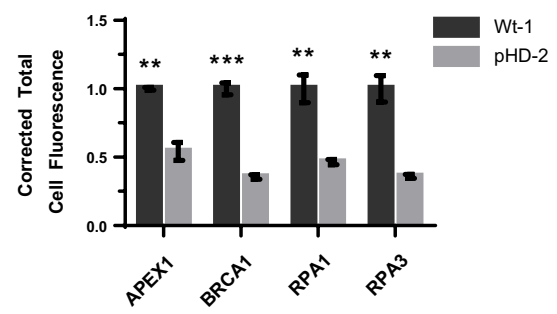

Figure 2 Down-regulation of DNA repair proteins in confirmed Huntington disease (HD) fibroblasts. A: Immunocytochemistry staining of APEX1, BRCA1, RPA1, and RPA3 in wild-type 1 (Wt-1), potential HD 1 (pHD-1), and pHD-2 cells. Differences in expression levels of each protein were determined by total fluorescence of cell area of pHD-1 (B) and pHD-2 (C) compared with Wt-1. Primary antibodies were counterstained with secondary conjugated Alexa Fluor 488. Nuclei staining was conducted with DAPI. Images were acquired using a Zeiss Axio microscope (Carl Zeiss AG, Jena, Germany) with a $10 \times$ objective. Error bars represent the SD of the mean corrected total cell fluorescence. Statistical significance was determined using a two-tailed, homoscedastic $t$-test. ${ }^{* *} P<0.01$, $* * * P<0.001$. region. Untreated pHD-1 was methylated at -437 and -28 $\mathrm{bp}$, whereas untreated pHD-2 was methylated at -435 , $-427,-417,-412,-138,-68,-28$, and -7 bp. Importantly, no methylated $\mathrm{CpG}$ sites were seen in pHD-1 or pHD-2 when treated with 5-azacytadine. Not only do these data confirm that 5-azacytidine treatments caused direct hypomethylation through passive cell replication, but this also brings to light the differential methylation patterns seen in HD fibroblasts. This differential methylation in the promoter region of APEXI elucidates a possible cause of gene down-regulation seen in our gene expression data.

\section{Intermittent 5-Azacytidine Treatments Induces TRN Stability in the HTT Gene}

Finally, we sought to determine whether the up-regulation of DNA repair genes by 5-azacytidine would stabilize TNR instability during long-term culture of HD cells. Previous studies have reported contradictory findings regarding global hypomethylation and its effect on TNR stability in various transgenic CAG repeat models ${ }^{13,30}$ We reasoned that the beneficial effect of 5-azacytidine treatment on DNA repair gene expression might also result in stabilization of the TNR region in HD-affected human cells. Cell lines pHD-1 and pHD-2 were plated in T-75 flasks and treated intermittently with $10 \mu \mathrm{mol} / \mathrm{L} 5$-azacytidine during 35 days to reach four population doublings, which in our experience is ample time to witness small expansions of TNR regions of the mutant $H T T$ gene. Genomic DNA samples were taken of pHD-1 and pHD-2 samples at the initial split before the treatment began and at the end of the 35-day time frame. In parallel to the treated samples, we passaged a subpopulation of each sample as a nontreated control. The pHD-1 sample began the treatment regimen with the HTT gene-affected allele containing 43 CAG repeats, with a small population of cells showing expansions in the affected allele up to 44 and 45 CAG repeats (Figure 5). At the end of the treatment schedule, the untreated control pHD-1 sample indicated the expected TNR expansion with the predominant population containing an allele with 44 CAG repeats and a small population of 45 CAG repeats. Conversely, the pHD-1 sample treated intermittently with 5azacytidine revealed TNR stability because it maintained a predominant population that contained an affected allele with $43 \mathrm{CAG}$ repeats, with a small population of $44 \mathrm{CAG}$ repeats. 

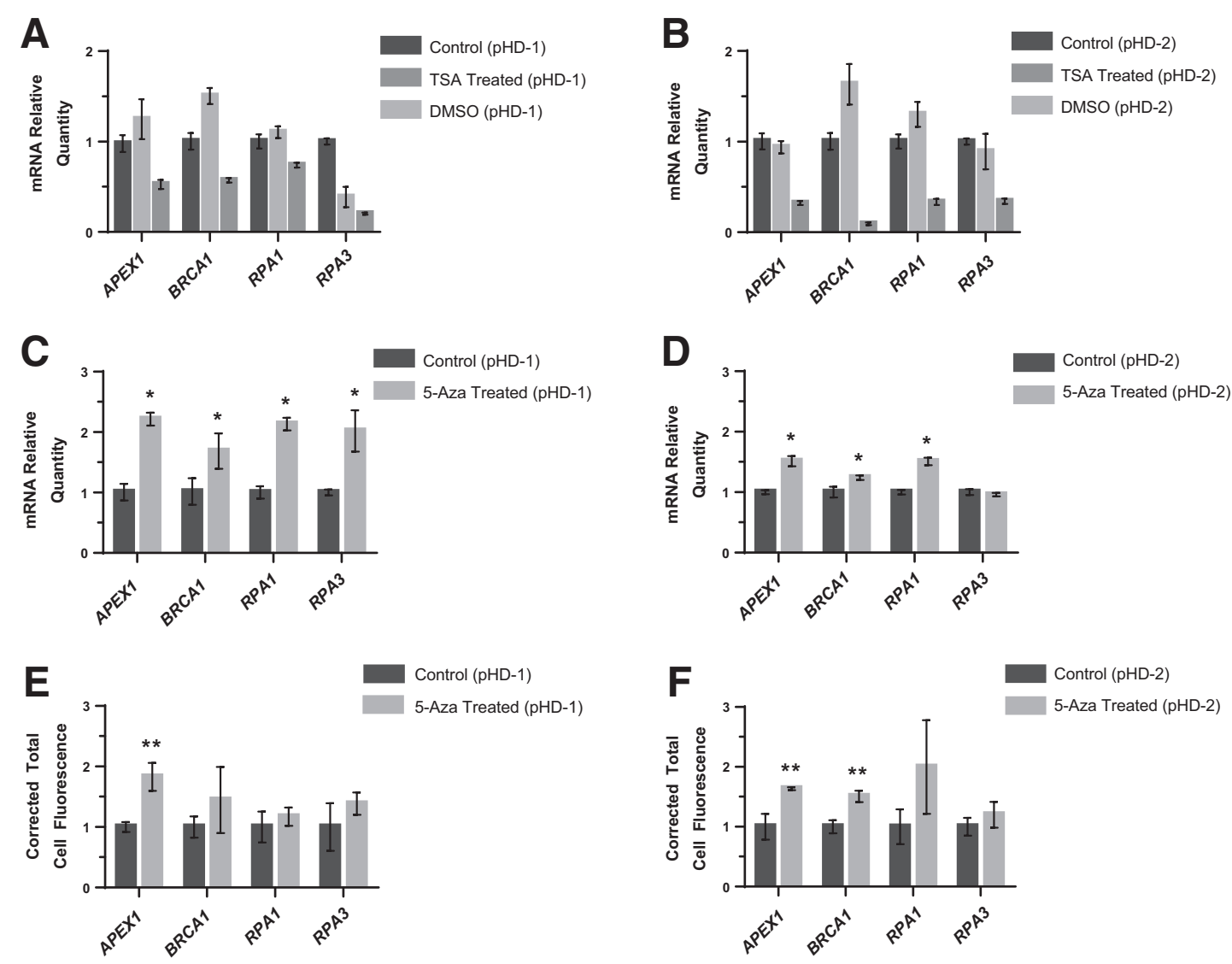

Figure 3 5-Azacytodine (5-Aza) but not trichostatin-A (TSA) increases DNA repair gene expression in Huntington disease (HD) fibroblasts. Potential HD 1 (pHD-1) (A) and 2 (pHD-2) (B) fibroblasts were treated with $5 \mu \mathrm{mol} / \mathrm{L}$ TSA for 24 hours, and gene expression levels of APEX1, BRCA1, RPA1, and RPA3 were analyzed and compared with cells treated with vehicle alone. Significant decreases were seen for all genes analyzed. pHD-1 (C) and pHD-2 (D) fibroblasts were treated with 10 $\mu \mathrm{mol} / \mathrm{L}$ 5-Aza for 120 hours, and gene expression was measured relative to cells treated with vehicle alone. Each tested gene has significant increases in expression for the pHD-1-treated fibroblast line. APEX1, BRCA1, and RPA1 have statistically significant increases in pHD-2 treated cells. $\mathbf{E}$ and $\mathbf{F}$ : Protein quantity was determined using corrected total cell fluorescence of the collected cell area using immunocytochemistry. E: Analysis reveals statistically significant up-regulation of APEX1 for 5-Aza-treated pHD-1 fibroblasts. F: pHD-2 fibroblasts treated with 5-Aza have statistically significant up-regulation of APEX1 and BRCA1. Bars represent the mean corrected total cell fluorescence (CTCF) of at least three images in random fields of view. Error bars represent the SD of the mean CTCF of each image. CTCF is relative to the untreated sample of each fibroblast line. ${ }^{*} P<0.05,{ }^{*} P<0.01$. DMS0, dimethyl sulfoxide.

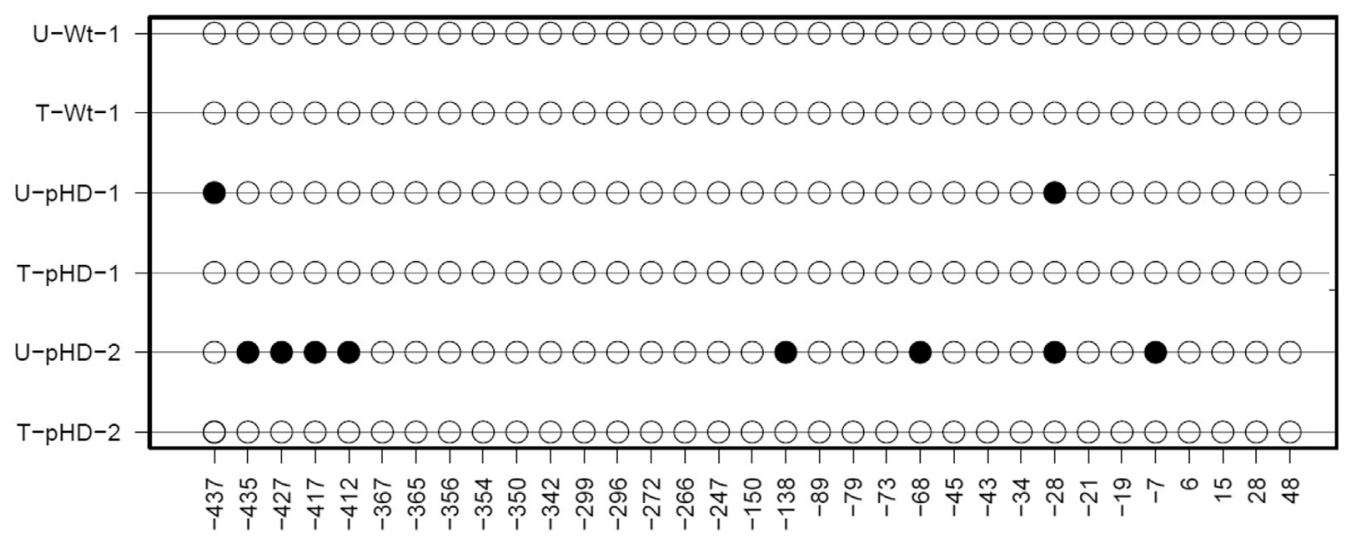

Figure 4 Differential methylation of APEX1 promoter region of Huntington disease (HD) and wild-type (Wt) cells. Genomic DNA was isolated from untreated Wt-1 (U-Wt-1), potential HD-1 (U-pHD-1), and potential HD-2 (U-pHD2) cells. Bisulfite sequencing was used to analyze the promoter region of APEX1. Open and closed circles represent unmethylated and methylated CpGs, respectively. U-Wt-1 has no methylated CpG sites in the promoter region, whereas U-pHD$1(-437$ and -28 bp) and U-pHD-2 ( $-435,-427,-417,-138,-68,-28$, and -7 bp) contain methylated CpGs. Treatment of each cell line with $10 \mu$ mol/L 5 -azacytadine for 120 hours (T-Wt-1, T-pHD-1, an pHD-2, respectively) results in loss of all methylated CpG sites in T-pHD-1 and T-pHD-2, whereas T-Wt-1 remains unmethlyated at all sites. 
pHD-1 Affected Allele

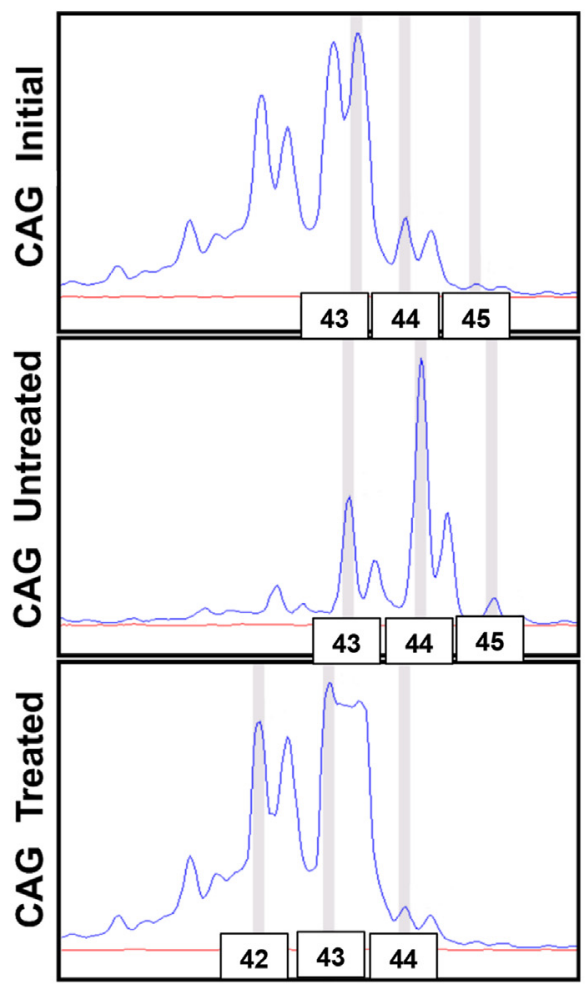

pHD-2 Affected Allele

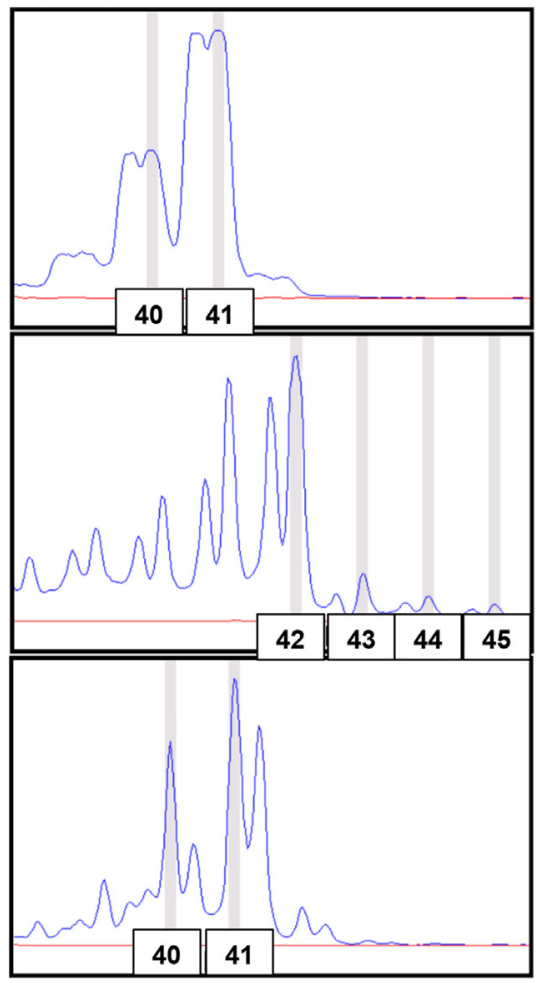

Figure 5 5-Azacytidine treatment stabilizes trinucleotide repeats (TNRs) of mitotically active Huntington disease (HD) fibroblasts. Two HD fibroblast cell lines were treated with $10 \mu \mathrm{mol} / \mathrm{L} \mathrm{5-}$ azacytidine intermittently in 35 days. The top panels represents the cytosine-adenine-guanine (CAG) expansions at treatment day 0 . The middle panels represents the CAG expansion of the untreated control at day 35 . The bottom panels show the CAG expansion of the 5-azacytidine treated samples after 35 days. Potential HD 1 (pHD-1) fibroblasts have beginning and treated samples with CAG repeats primarily of 43 , with a small population of cells having expansions up to 44 to 45 . Untreated pHD-1 has a CAG expansion of predominantly 44, with a small population of 45 . pHD-2 fibroblasts have beginning and treated samples with CAG repeats of predominantly 41 . Untreated pHD-2 have expansions of predominantly 42 but with small subpopulations expanding up to 45 .
Similar results were witnessed in sample pHD-2, where initial expansion of the affected allele was characterized as having predominantly $41 \mathrm{CAG}$ repeats (Figure 5). At the end of the treatment cycles, the untreated pHD-2 sample had predominantly $42 \mathrm{CAG}$ repeats with instability within the population up to 45. Again, conversely, the treated pHD-2 sample revealed that most cells had maintained $41 \mathrm{CAG}$ repeats, with only a small subset of the cell population having 42 CAGs. Interestingly, these data indicate that the normal expansion of CAG repeats during culture of HD cells is mitigated by 5-azacytidine treatment and is a proof of principle that pharmacologic intervention can be used to prevent TNR instability in HD.

\section{Discussion}

To the best of our knowledge, this is the first report demonstrating down-regulation of DNA repair genes in multiple proliferating HD cell lines. Down-regulation of APEX1, BRCA1, RPA1, and RPA3 mRNA levels were so consistent, we were able to use them to predict the HD status of two unknown samples. We pursued these genes in particular because they are implicated in TNR contractions/expansions and were found to be downregulated in a preliminary screen of DNA repair gene expression in HD cells (unpublished data). ${ }^{24-29}$ We found that this down-regulation is likely mediated, at least in part, by DNA methylation because the DNA methyltransferase inhibitor 5-azatacytadine can partially restore DNA repair expression. Perhaps most strikingly, 5-azacytadine treatment stabilized TNRs during long-term culture of proliferating HD cells. These results offer a novel insight into HD pathogenesis and identify new potential biomarkers and therapeutic strategies.

Although there is overwhelming evidence indicating that HD and DNA repair are associated, no comprehensive model has been determined to clearly explain TNR instability in HD. Importantly, the identification of APEX1, BRCA1, RPA1, and, RPA3 down-regulation in HD can potentially provide therapeutic targets and biomarkers for disease progression or treatment success. These genes obviously represent a small subset of all DNA repair genes likely involved in TNR instability, and thus future studies will expand on these findings to determine whether other genes are similarly affected in HD.

The $\mathrm{mHtt}$ protein produced by the expanded TNR region of exon 1 in the HTT gene interacts nonspecifically with many unintended targets. The resulting interaction leads to cellular dysfunction on multiple levels, including transcriptional dysregulation, mitochondrial dysfunction, altered vesicular transport, reactive oxygen species defense, abnormal endocytosis and secretion, and increased apoptosis. The elongated gene encodes for repetitive glutamine amino acids (polyglutamine [poly-Q]), in which these poly-Q chains contain hydrophilic properties. The $\mathrm{mHtt}$ protein exhibits a toxic gain of function in which poly-Q chains bind unintended targets, sequestering them from normal function. ${ }^{31}$ Consequently, expanded poly-Q regions of $\mathrm{mHtt}$ disrupt normal cell functions through interaction with multiple basal transcription factors, causing gene expression dysregulation, including 
those encoding CREB, CREBBP, Sp1, TFII30, NRSF/REST, PFC1 $\alpha$, BCL11b, TBP, and others. ${ }^{32-37}$ Furthermore, extensive changes in DNA methylation are associated with the expression of $\mathrm{mHtt}$. Interestingly, these changes in methylation patterns seem to be sequence specific to DNA-binding motifs. ${ }^{38}$ Therefore, it seems likely that $\mathrm{mHtt}$ might disrupt normal expression of APEX1, BRCA1, RPA1, and RPA3 through both disruption of relevant transcription factors and methylation patterning. Our observations revealing intermittent treatment of 5-azacytidne and its up-regulation of DNA repair genes further support the finding that $\mathrm{mHtt}$ might be interfering with DNA methylation epigenetics. Any dysfunction of methylation pattern maintenance caused by $\mathrm{mHtt}$ would clearly alter physiologic epigenetics and would have repercussions long before HD neuronal dysfunction presents, especially when considering the extensive involvement that methylation plays on neurogenesis. ${ }^{39-41}$ Although the passive demethylation by cell division does not address mitotically inactive disease-associated cells (medium spiny neurons), the stabilization of the CAG expansion in exon 1 of the HTT gene over four population doublings indicates the possibility that controlling TNR instability could prevent HD-associated genetic anticipation. Furthermore, our finding that the DNA repair expression of APEX1 did not return to wild-type levels despite complete promoter hypomethylation suggests additional mechanisms (ie, direct transcriptional disruption) factor into the DNA repair gene down-regulation.

Whatever the mechanism, it is clear that mitotically active HD cells have reduced DNA repair gene expression. Future studies should seek to answer key questions raised in this study. First, we are attempting to identify whether this down-regulation persists in mitotically inactive neurons and whether DNA repair deficiencies lead to increased transcription-mediated TNR instability in neurons as well. Furthermore, because RT-qPCR is a relatively quick assay compared with current sequencing and triplet repeat primed PCR technologies, the utility of gene expression as a biomarker for HD disease should be explored. Finally, the stabilization of CAG repeats within the HD gene by 5azacytadine-induced up-regulation of DNA repair genes is intriguing. Unfortunately, 5-azacytadine is likely not a viable preventive therapeutic option, but the data here are a proof of principle that pharmacologic interventions aimed at up-regulated DNA repair mechanisms during development could delay onset and severity of the disease by preventing CAG expansions in mutant HTT. As such, future work should look to identify other molecules that have a similar effect on DNA repair gene expression and TNR stability and can be administered for long periods in young patients with a mutant $H T T$ allele.

DNA repair genes APEXI, BRCA1, RPAl, and RPA3 are significantly down-regulated in mitotically active HD fibroblasts. Gene expression levels of these four genes accurately predicted the HD status of two unknown samples. Furthermore, the suppression of DNA repair gene expression is mediated, in part, by DNA methylation and can be partially restored by treatment with 5-azacytadine. Finally, 5-azacytadine treatment prevented TNR expansion during long-term culture of HD cells. We conclude that downregulation of DNA repair genes leads to increased TNR instability in HD cells, and restoration of normal DNA repair expression can reduce TNR instability in the HTT gene.

\section{Acknowledgments}

We thank Mohammed Jamma (ARUP Laboratories) for his assistance with the design of the macroinstructions for triplet repeat primed PCR analysis and Dr. Tabetha Sundin (Sentara Norfolk General Hospital) for her troubleshooting expertise and donation of supplies.

\section{Supplemental Data}

Supplemental material for this article can be found at http://dx.doi.org/10.1016/j.ajpath.2016.03.014.

\section{References}

1. MacDonald ME, Ambrose CM, Duyao MP, Myers RH, Lin C, Srinidhi L, Barnes G, Taylor SA, James M, Groot N, MacFarlane H, Jenkins B, Anderson MA, Wexler NS, Gusella JF; Huntington's Disease Collaborative Research Group: A novel gene containing a trinucleotide repeat that is expanded and unstable on Huntington's disease chromosomes. Cell 1993, 72:971-983

2. Bates G: Huntingtin aggregation and toxicity in Huntington's disease Lancet 2003, 361:1642-1644

3. Ranen NG, Stine OC, Abbott MH, Sherr M, Codori AM, Franz ML, Chao NI, Chung AS, Pleasant N, Callahan C, Kasch LM, Ghaffari M, Chase GA, Kazazian HH, Brandt J, Folstein SE, Ross CA: Anticipation and instability of IT-15 (CAG)n repeats in parent-offspring pairs with Huntington disease. Am J Hum Genet 1995, 57:593-602

4. Langbehn DR, Brinkman RR, Falush D, Paulsen JS, Hayden MR; International Huntington's Disease Collaborative Group: A new model for prediction of the age of onset and penetrance for Huntington's disease based on CAG length. Clin Genet 2004, 65:267-277

5. Langbehn DR, Hayden MR, Paulsen JS; PREDICT-HD Investigators of the Huntington Study Group: CAG-repeat length and the age of onset in Huntington disease (HD): a review and validation study of statistical approaches. Am J Med Genet B Neuropsychiatr Genet 2010, 153B:397-408

6. Lee JM, Ramos EM, Lee JH, Gillis T, Mysore JS, Hayden MR, et al: CAG repeat expansion in Huntington disease determines age at onset in a fully dominant fashion. Neurology 2012, 78:690-695

7. Walker FO: Huntington's Disease. Semin Neurol 2007, 27:143-150

8. Gomes-Pereira M, Fortune MT, Monckton DG: Mouse tissue culture models of unstable triplet repeats: in vitro selection for larger alleles, mutational expansion bias and tissue specificity, but no association with cell division rates. Hum Mol Genet 2001, 10:845-854

9. Fortune MT, Vassilopoulos C, Coolbaugh MI, Siciliano MJ, Monckton DG: Dramatic, expansion-biased, age-dependent, tissuespecific somatic mosaicism in a transgenic mouse model of triplet repeat instability. Hum Mol Genet 2000, 9:439-445

10. Telenius H, Kremer B, Goldberg YP, Theilmann J, Andrew SE, Zeisler J, Adam S, Greenberg C, Ives EJ, Clarke LA, Hayden MR: Somatic and gonadal mosaicism of the Huntington disease gene CAG repeat in brain and sperm. Nat Genet 1994, 6:409-414 
11. Kennedy L, Shelbourne PF: Dramatic mutation instability in HD mouse striatum: does polyglutamine load contribute to cell-specific vulnerability in Huntington's disease? Hum Mol Genet 2000, 9: 2539-2544

12. Kovalenko M, Dragileva E, St Claire J, Gillis T, Guide JR, New J, Dong H, Kucherlapati R, Kucherlapati MH, Ehrlich ME, Lee JM, Wheeler VC: Msh2 acts in medium-spiny striatal neurons as an enhancer of CAG instability and mutant huntingtin phenotypes in Huntington's disease knock-in mice. PLoS One 2012, $7: \mathrm{e} 44273$

13. Gomes-Pereira M, Monckton DG: Chemically induced increases and decreases in the rate of expansion of a CAG*CTG triplet repeat. Nucleic Acids Res 2004, 32:2865-2872

14. Chatterjee N, Lin Y, Santillan BA, Yotnda P, Wilson JH: Environmental stress induces trinucleotide repeat mutagenesis in human cells. Proc Natl Acad Sci U S A 2015, 112:3764-3769

15. Wheeler VC, Persichetti F, McNeil SM, Mysore JS, Mysore SS, MacDonald ME, Myers RH, Gusella JF, Wexler NS; Group US-VCR: Factors associated with HD CAG repeat instability in Huntington disease. J Med Genet 2007, 44:695-701

16. Huang B, Wei W, Wang G, Gaertig MA, Feng Y, Wang W, Li XJ, Li S: Mutant huntingtin downregulates myelin regulatory factormediated myelin gene expression and affects mature oligodendrocytes. Neuron 2015, 85:1212-1226

17. Khakh BS, Sofroniew MV: Astrocytes and Huntington's disease. ACS Chem Neurosci 2014, 5:494-496

18. Chan CS, Surmeier DJ: Astrocytes go awry in Huntington's disease. Nat Neurosci 2014, 17:641-642

19. Lee DY, McMurray CT: Trinucleotide expansion in disease: why is there a length threshold? Curr Opin Genet Dev 2014, 26:131-140

20. Sinden RR: Neurodegenerative diseases: origins of instability. Nature 2001, 411:757-758

21. Jama M, Millson A, Miller CE, Lyon E: Triplet repeat primed PCR simplifies testing for Huntington disease. J Mol Diagn 2013, 15: 255-262

22. Li LC, Dahiya R: MethPrimer: designing primers for methylation PCRs. Bioinformatics 2002, 18:1427-1431

23. Mallona I, Diez-Villanueva A, Peinado MA: Methylation plotter: a web tool for dynamic visualization of DNA methylation data. Source Code Biol Med 2014, 9:11

24. Beaver JM, Lai Y, Xu M, Casin AH, Laverde EE, Liu Y: AP endonuclease 1 prevents trinucleotide repeat expansion via a novel mechanism during base excision repair. Nucleic Acids Res 2015, 43: $5948-5960$

25. Li M, Volker J, Breslauer KJ, Wilson DM 3rd: APE1 incision activity at abasic sites in tandem repeat sequences. J Mol Biol 2014, 426: 2183-2198

26. Mason AG, Tome S, Simard JP, Libby RT, Bammler TK, Beyer RP, Morton AJ, Pearson CE, La Spada AR: Expression levels of DNA replication and repair genes predict regional somatic repeat instability in the brain but are not altered by polyglutamine disease protein expression or age. Hum Mol Genet 2014, 23:1606-1618
27. Andreoni F, Darmon E, Poon WC, Leach DR: Overexpression of the single-stranded DNA-binding protein (SSB) stabilises $\mathrm{CAG}^{*} \mathrm{CTG}$ triplet repeats in an orientation dependent manner. FEBS Lett 2010, 584:153-158

28. Broderick S, Rehmet K, Concannon C, Nasheuer HP: Eukaryotic single-stranded DNA binding proteins: central factors in genome stability. Subcell Biochem 2010, 50:143-163

29. Spiro C, McMurray CT: Nuclease-deficient FEN-1 blocks Rad51/BRCA1-mediated repair and causes trinucleotide repeat instability. Mol Cell Biol 2003, 23:6063-6074

30. Gorbunova V, Seluanov A, Mittelman D, Wilson JH: Genome-wide demethylation destabilizes CTG.CAG trinucleotide repeats in mammalian cells. Hum Mol Genet 2004, 13:2979-2989

31. Nucifora FC Jr, Sasaki M, Peters MF, Huang H, Cooper JK, Yamada M, Takahashi H, Tsuji S, Troncoso J, Dawson VL, Dawson TM, Ross CA: Interference by huntingtin and atrophin-1 with cbp-mediated transcription leading to cellular toxicity. Science 2001, 291:2423-2428

32. Dunah AW, Jeong H, Griffin A, Kim YM, Standaert DG, Hersch SM, Mouradian MM, Young AB, Tanese N, Krainc D: Sp1 and TAFII130 transcriptional activity disrupted in early Huntington's disease. Science 2002, 296:2238-2243

33. Zhai W, Jeong H, Cui L, Krainc D, Tjian R: In vitro analysis of huntingtin-mediated transcriptional repression reveals multiple transcription factor targets. Cell 2005, 123:1241-1253

34. Kazantsev A, Preisinger E, Dranovsky A, Goldgaber D, Housman D: Insoluble detergent-resistant aggregates form between pathological and nonpathological lengths of polyglutamine in mammalian cells. Proc Natl Acad Sci U S A 1999, 96:11404-11409

35. Steffan JS, Kazantsev A, Spasic-Boskovic O, Greenwald M, Zhu YZ, Gohler H, Wanker EE, Bates GP, Housman DE, Thompson LM: The Huntington's disease protein interacts with p53 and CREB-binding protein and represses transcription. Proc Natl Acad Sci U S A 2000, 97:6763-6768

36. Li SH, Cheng AL, Zhou H, Lam S, Rao M, Li H, Li XJ: Interaction of Huntington disease protein with transcriptional activator Sp1. Mol Cell Biol 2002, 22:1277-1287

37. Seredenina T, Luthi-Carter R: What have we learned from gene expression profiles in Huntington's disease? Neurobiol Dis 2012, 45: 83-98

38. Ng CW, Yildirim F, Yap YS, Dalin S, Matthews BJ, Velez PJ, Labadorf A, Housman DE, Fraenkel E: Extensive changes in DNA methylation are associated with expression of mutant huntingtin. Proc Natl Acad Sci U S A 2013, 110:2354-2359

39. Covic M, Karaca E, Lie DC: Epigenetic regulation of neurogenesis in the adult hippocampus. Heredity 2010, 105:122-134

40. Sun J, Sun J, Ming GL, Song H: Epigenetic regulation of neurogenesis in the adult mammalian brain. Eur J Neurosci 2011, 33 : 1087-1093

41. Szulwach KE, Li X, Smrt RD, Li Y, Luo Y, Lin L, Santistevan NJ, Li W, Zhao X, Jin P: Cross talk between microRNA and epigenetic regulation in adult neurogenesis. J Cell Biol 2010, 189:127-141 